\title{
Das Globalbudget steht an der Hintertür
}

\author{
Jürg Schlup \\ Dr. med., Präsident der FMH
}

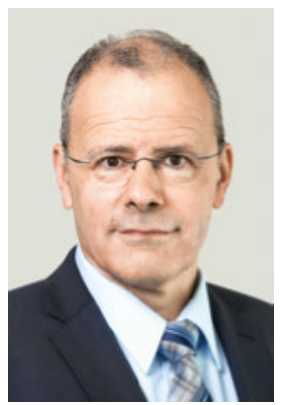

Die vom Eidgenössischen Departement des Innern (EDI) beauftragte Expertise zu Kostendämpfungsmassnahmen [1] wurde bislang vor allem wegen ihres Vorschlags einer kollektiven Kostenobergrenze für Patientenbehandlungen diskutiert. Trotz dieser Gefahr für unsere Gesundheitsversorgung gilt es aber auch, die 37 anderen Massnahmen des Berichts nicht aus dem Blick zu verlieren. Im Februar 2018 stellte die FMH bereits eigene Vorschläge kostendämpfender Massnahmen vor, die teilweise mit denen der Experten überlap-

\section{Als Mediziner müssen wir vor Heils- versprechen warnen, die viel Wirkung ohne Nebenwirkung in Aussicht stellen.}

pen [2]. Offen blieb bislang, wie das weitere Vorgehen zur Umsetzung der vielen in der Expertise vorgeschlagenen Massnahmen aussehen wird.

Literatur

1 Bericht der Expertengruppe. Kostendämpfungsmassnahmen zur Entlastung der obligatorischen Krankenpflegeversicherung. 24. August 2017.

2 Wille N, Glarner I Schlup J. Die FMH zur Dämpfung des Kostenanstiegs im Gesundheitswesen. Schweiz Ärztezeitung. 2018;99(8):224-6.

3 Eidgenössisches Depar tement des Innern EDI/Bundesamt für Gesundheit BAG. Kostendämpfungsmass nahmen zur Entlastung der obligatorischen Krankenpflegeversicherung (OK): Erste Massnahmen, die geprüft werden. 29.3.2018

4 Wille N, Glarner J, Schlup J Die FMH zum ersten Kostendämpfungspaket des Bundesrats. Schweiz Ärztezeitung. 2018;99(19-20): 604-6.
Nun hat der Bundesrat am 28. März 2018 neun der 38 Massnahmen priorisiert sowie drei zusätzlichen Vorschlägen des EDI Priorität eingeräumt [3]. Ab Seite 604 stellen wir die Positionen der FMH zu diesen Massnahmen vor [4]. Wir begrüssen besonders das Vorhaben, die Governance-Konflikte der Kantone zu reduzieren. Ebenfalls begrüsst die FMH Verbesserungen der Rechnungskontrolle. Auch der bundesrätlich priorisierte Experimentierartikel könnte aus Sicht der FMH einen positiven Beitrag zur Entwicklung des Gesundheitswesens leisten, sofern man die Leistungserbringer einbezieht und die Indikatoren zur Evaluation von Pilotprojekten gemeinsam und transparent festlegt. Leider werden die Leistungserbringer aber ausgeklammert, und auch die fünf auf den ambulanten Tarif bezogenen Massnahmen schnüren uns ein immer engeres Korsett, während der Bund weitreichende Kompetenzen z.B. als "Anpassungs- und Festlegungsbehörde» ([1], S. 73) anstrebt. Weil niemand die schwierigen Baustellen im Tarifbereich besser kennt als die FMH, begrüssen wir alle Neuerungen, die Verbesserungen erwarten lassen. Immer mehr staatliche
Kompetenzen - auch subsidiäre - sind aber kontraproduktiv, weil sie die Tarifpartnerschaft unterlaufen. Bereits heute hebeln sie die Tarifautonomie nahezu aus, indem sie ein Scheitern von Verhandlungen für einzelne Beteiligte sehr attraktiv machen. Möchte man also nicht bewusst einem Staatstarif den Weg bereiten, müssen die angedachten Reformen unter dem Vorzeichen der Struktur- und Organisationsautonomie der Tarifpartner stehen.

Besonders kritisch ist vor diesem Hintergrund der vom Bundesrat priorisierte Vorschlag des EDI einer «Steuerung der Kosten durch die Tarifpartner» [3]. Die geforderte gesetzliche Verpflichtung, in den "Tarifverträgen Massnahmen zur Steuerung der Kosten und Leistungen vorzusehen", um "das Kostenwachstum zu bremsen" [3] - wiederum mit subsidiärer Kompetenz von Bund oder Kantonen -, ist ein Versuch, das Globalbudget durch die Hintertür einzuführen: Zukünftig wäre die unzureichende Steuerung der Tarifpartner verantwortlich, wenn Gesundheitsleistungen in den Augen der Politik zu teuer wären - und der Staat würde übernehmen. Wir werden uns jedoch nicht die Aufgabe zuschieben lassen, die Patientenversorgung gemäss politischen Kostenkriterien zu «steuern» faktisch also einzuschränken -, und halten diese Hintertür verschlossen.

\section{Wir lassen uns nicht die Aufgabe zuschieben,} die Patientenversorgung einzuschränken.

Ob die verschlossene Hintertür allerdings nutzt, wenn die CVP und der Bund bereits mit dem Rammbock vor dem Haupteingang stehen, ist fraglich. Dem Wähler Kostendämpfung ohne spürbare Abstriche und bei sogar verbesserter Qualität zu versprechen, gilt offenbar aktuell als politisches Erfolgsrezept. Als Mediziner müssen wir aber vor Heilsversprechen warnen, die viel Wirkung ohne Nebenwirkung in Aussicht stellen. Diese unpopuläre Aufgabe werden wir gewissenhaft wahrnehmen. 